\title{
Method of estimating the maximum penetration level of wind power using transient frequency deviation index based on COI frequency
}

\begin{abstract}
Among the significant renewable resources, wind power has acquired the spotlight, due to the quick development in wind energy conversion technology. However, the penetration level of wind power cannot be increased randomly for maximum power generation primarily because the wind farms cannot always provide a precise amount of power similar to the conventional synchronous generators. Since the frequency is the most affected by the penetration of wind power due to the variable nature and the less inertia of these turbines, it is necessary to estimate the maximum level of wind energy that can maintain a nominal frequency and does not affect the frequency stability of the power system. In this paper, new procedures to determine the maximum wind penetration level using transient frequency deviation index (TFDI) and based on the center of inertia frequency are presented. The results of procedures are established through the dynamic simulation of WSCC 9-bus test system. By using this method, the critical wind level can be assessed while the system preserves acceptable operation frequency.
\end{abstract}

Keyword: Penetration of wind power; Frequency deviation; Center of inertia frequency; Frequency trajectory 